\title{
Clinical features, visual outcome and association with systemic disease in patients with episleritis and scleritis. A study of 50 eyes
}

\author{
Mohamed AI ${ }^{1}$, Annamalai R ${ }^{2}$, Muthayya $\mathbf{M}^{3}$ \\ ${ }^{1}$ Dr. Mohamed Anjum Iqbal, Postgraduate in Ophthalmology, ${ }^{2}$ Dr Radha Annamalai, Associate Professor of \\ Ophthalmology, ${ }^{3}$ Prof. Muthayya Muthukumar, Senior Consultant in Ophthalmology. All are affiliated with Sri \\ Ramachandra University, Chennai, India.
}

Address for correspondence: Dr. RadhaAnnamalai, Email: drradhaannamalai@ yahoo.co.in

\begin{abstract}
Introduction: Episcleritis and scleritis are inflammatory disorders which can have an acute presentation and are frequently encountered by primary care practitioners.Episcleritis is a benign, self-limited disease that resolves within 2 to 3 weeks. Scleritis is painful, causing visual loss due to uveitis, glaucoma, cataract, and retinal detachment. Both are associated with underlying systemic diseases.We aim to report clinical features, visual outcome and association with systemic disease in patients with visual symptoms. Methods: This is a prospective cohort study conducted on 50 patients over 1 year in a tertiary eye care hospital Complete ophthalmic evaluation was performed. Patients were followed up for one year to look for response to treatment and development of complications. Results: In our study, the incidence of episcleritis was more frequent than scleritis. The age group affected was 34 to 52 years and more in women. Most of our patients with episcleritis showed resolution within 3 weeks. Scleritis was associated with decreased vision and more complications. In both forms of inflammation rheumatoid arthritis, systemic lupus erythematosus and psoriasis were the most common etiology. Conclusion: In rheumatoid arthritis, systemic lupus erythematosus and psoriasis patients, episcleritis and scleritis can be the initial clinical presentation. Clinical features are more severe in scleritis and can be diagnosedwith B scan ultrasound. Visual loss in both can be prevented by early treatment. We found the association with rheumatoid arthritis and SLEto be high in our population. Infections were not as common as autoimmune diseases in the population studied.
\end{abstract}

Key words: Episcleritis, Scleritis, Rheumatoid Arthritis, Uveitis

\section{Introduction}

Ocular involvement occurs commonly with systemic diseases and treatment approach needs to be directed towards the underlying aetiology. Involvement of the eye in such cases may be inflammatory, infectious, vascular, traumatic or idiopathic in nature. Progression of the disease and onset of sequelae can result in blindness and in several situations, the general physician is the first to see the patient. Episcleritis and scleritis are inflammatory disorders frequently encountered by primary care practitioners. They may extend to adjacent ocular tissues with blinding consequences and may be associated with immune mediated systemic disorders [1] or infections

Manuscript received $3^{\text {rd }}$ April 2016

Reviewed: $14^{\text {th }}$ April 2016

Author Corrected: $25^{\text {th }}$ April 2016

Accepted for Publication $6^{\text {th }}$ May 2016
[2]. Patients present with redness, pain and visual loss of varying severity. Episcleritis is a self-limited disease that usually resolves within 2 to 3 weeks. Scleritis is an inflammatory process of the sclera and adjacent tissues with varied forms of clinical presentations and comorbidities [3]. It is a painful, disease of the eye that is often associated with visual loss and systemic disease. Significant complications of scleritis are uveitis, glaucoma, cataract, and retinal detachment [4]. Anatomically it may involve the anterior or posterior segment. Anterior scleritis can be diffuse, nodular or necrotising. The necrotizing form of scleritis is the most serious and needs to be treated aggressively with non-steroidalanti-inflammatory (NSAIDS) drugs, systemic steroids and sometimes immunomodulatory drugs. Thenecrotising type can be with or without inflammation. Scleromalaciaperforansis 
associated with rheumatoid arthritis (RA) and is a serious form of necrotizing anterior scleritis. Posterior scleritis is less common but can cause severe complications and visual loss if not treated.

The clinical appearance of episcleritis may resemble scleritis but the inflammation and erythema are localised to the episcleral tissue. Recurrences are more frequent with episcleritis, it is sometimes associated with systemic diseases [5] but it does not cause severe or permanent visual loss. It is self-limited, benign and clinically classified as nodular and diffuse types. The nodular form is characterised by a discrete, elevated area of episclera.In the simple type, there is diffuse congestion due to involvement of anterior ciliary vessels. In routine practice, differentiation of episcleritis and scleritis can be made using $2.5 \%$ phenylephrine eye drops which blanches only the conjunctival and episcleral vessels but not the deeper scleral plexus. Hence if redness in a patient decreases or disappear then adiagnosis of episcleritis can be made.

\section{Materials and Method}

This study was performed on 50 patients over a period of 1 yearto evaluate the clinical experience with episcleritis and scleritis at a tertiary care eye center. It was performed in concurrence with the departments of rheumatology and internal medicine. The design was a prospective cohort study conducted on all new patients with episcleritis and scleritis who presented to the ophthalmology outpatient department with visual complaints. The study was conducted in a tertiary referral hospital in India. Prior to the study ethics committee clearance was obtained.Inclusion criteria were patients above 18 years of age with episcleritis or scleritis. Patients with prior cataract, glaucoma, immunosuppression and history of allergy to nonsteroidal anti-inflammatory drugs, were excluded from the study. The patients were divided into two study groups based on whether they had associated systemic diseases at presentation or not.

A complete ophthalmic evaluation was performed on all patients. After obtaining a detailed history, slit lamp examination, indirect ophthalmoscopy, biomicroscopy tonometry and refraction was done. B scan ultrasound was done on patients whenever scleritis was suspected. We investigated all patients of episcleritis and scleritis. Laboratory workup comprised of complete blood count, erythrocyte sedimentation rate (ESR), rheumatoid factor, antinuclear antibody, VDRL/FTA-ABS, serum uric acid, urinalysis, Mantoux testand a chest x-ray. However in some patients, based on clinical suspicion, further blood specific tests for herpes simplex, herpes zoster, sarcoidosis and vasculitis such as polymerase chain reaction (PCR) of blood for herpes virus, $\mathrm{P}$ ANCA, C-ANCA, angiotensin converting enzyme and serum lysozyme assay was done

Patients were followed up for atleast one year and during each visit a clinical examination and relevant investigations were performed. During active disease, weekly reviews were done for one month. After response to treatment or resolution, monthly reviews for one year were performed to look for complications and visual improvement.Blood workup including complete blood count, liver function, renal function and blood sugar was done when treatment with oral steroids (1mg/kg bodyweight/ day) or immunosuppressive drugs were planned. Immunosuppressive drugs used in our study were tabletmethotrexate $7.5 \mathrm{mg}$ weekly, tablet mycophenolate mofetill gm per day and tablet azathioprine 1 - $2 \mathrm{mg} / \mathrm{kg}$ / day.

\section{Results}

Fifty patients with episcleritis and scleritis were seen over a one year period. Among this cohort, 38 patients had episcleritis and 12 had scleritis. Episcleritis and scleritis were bilateral in $18 \%$ and $9 \%$ respectively $(p=0.004)$. The age range at presentation was maximum between 34 to 52 years. $81 \%$ were female. Associated systemic disease was seen in $63 \%$. It was rheumatoid arthritis (RA) in $36 \%$, systemic lupus erythematosus (SLE) in 18\%, tuberculosis in $9 \%$ and psoriasis in $2 \%$. Redness was the presenting feature in $98 \%$ of episcleritis and $21 \%$ of scleritis. On initial visit, decreased vision was noted in $6 \%$ of episcleritis and $43 \%$ of scleritis. Pain was seen in $74 \%$ of episcleritis and $82 \%$ of scleritis. Ocular complications that we noted were cataracts, glaucoma and exudative retinal detachment. None of our episcleritis patients had a decrease in visual acuity as against $21 \%$ of scleritis patients who did. Ocular complications occurred in 3 patients with episcleritis but in 12 patients with scleritis $(p=0.002)$. Necrotising scleritis and posterior scleritis were more often associated with complications. Incidence of complications was much more in necrotizing scleritis and posterior scleritis than nodular or diffuse anterior scleritis. All patients with episcleritis responded to topical NSAID along with lubricant eye drops. Only $3 \%$ of patients with episcleritis required oral NSAIDS and none of our episcleritis patients required steroids for treatment. In patients with scleritis, $21.3 \%$ required NSAID, $60 \%$ required oral prednisolone 
and $12 \%$ immunosuppressive drugs. Patients with necrotizing and posterior scleritis (33\% and $21 \%$ respectively)more often needed immunosuppressive drugs than diffuse and nodular scleritis who responded with NSAIDS(1\% and $3 \%$ respectively) $\mathrm{p}=0.0003$. The follow- up was for 1 year. We noted recurrence in 3 patients with episcleritis but no recurrence in scleritis. Even the recurrences settled with topical NSAIDS for two weeks.

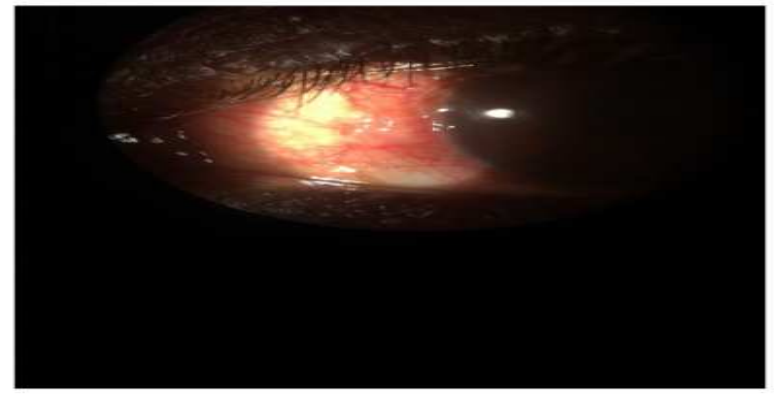

Figure-1: Nodular episcleritis.

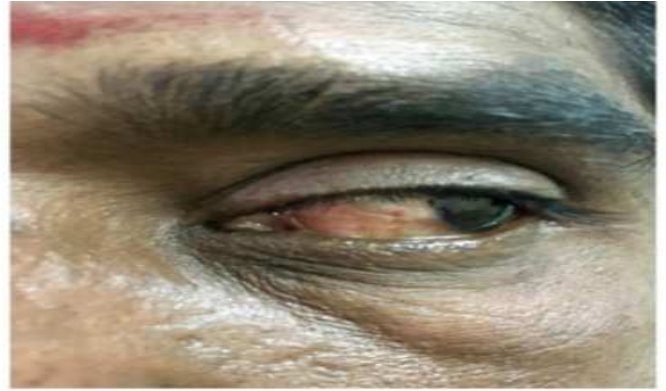

Figure -2: Sectoral congestion in scleritis.

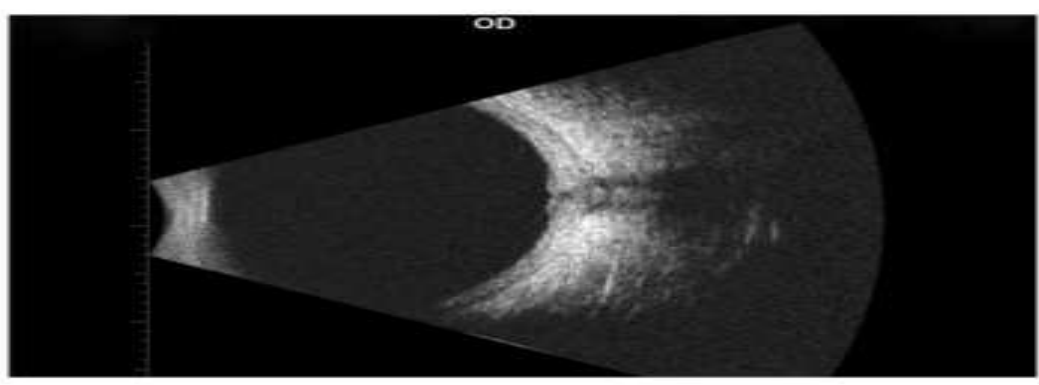

Figure- 3: B scan ultrasound of eye showing scleritis.

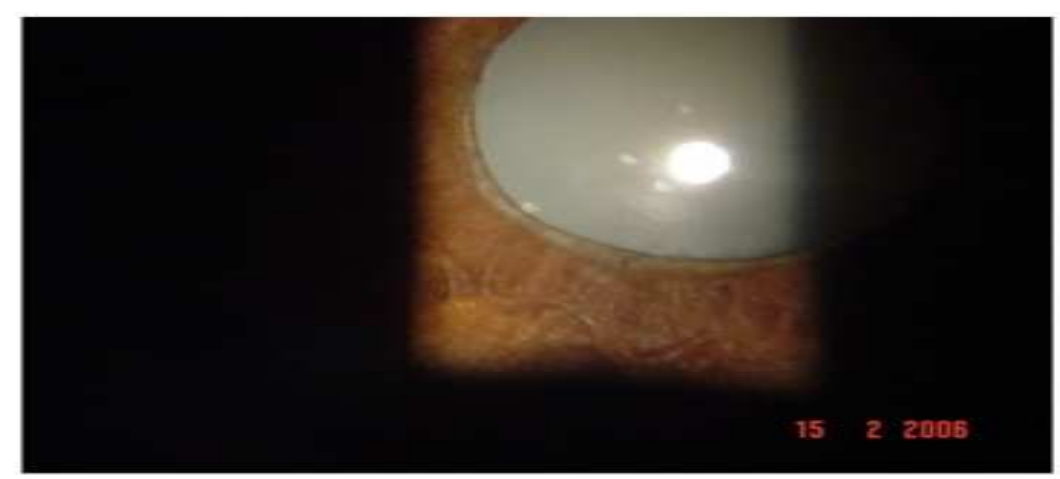

Figure -4: Steroid induced cataract following scleritis treatment.

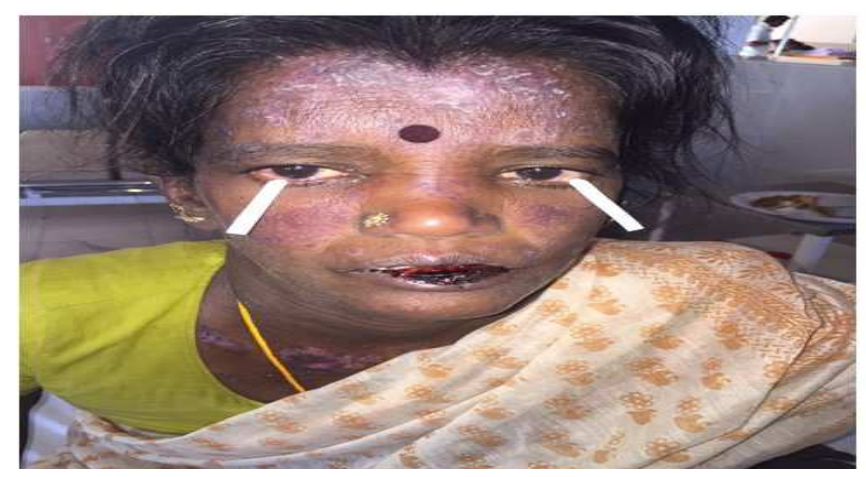

Figure-5: Systemic lupus erythematosus with scleritis and dry eye. 


\section{Discussion}

The study was performed over a one year period to evaluate episcleritis and scleritis at a tertiary care eye centre and to identify clinical features in them.Episcleritis is known to be a disease which is benign in nature as against scleritis which has a potential to cause vision loss and severe complications [6]. In our study we found that episcleritis is associated with systemic diseases in the majority of patients and RA,SLE and psoriasis were the most common aetiology. Patients with episcleritis resolve completely with a combination of topical and systemic nonsteroidal anti-inflammatory drugs. We did not use steroids in any of the patients in this group. In the scleritisgroup treatment was done after investigating for possible underlying infections such as tuberculosis, syphilis or herpes simplex and zoster. After ruling out infection management was done using oral steroids in the dose of $1 \mathrm{mg} / \mathrm{kg}$ body weight. Depending on the response of the patient immunosuppressives were added once a week.In some situations when the disease progresses rapidly or causes severe vision loss more potent drugs to control inflammation are required. In the paediatric population, some children present with posterior scleritis having very poor vision, retinal striae and disc oedema and require immediate systemic steroids and immunosuppression. If intervention is done early prognosis for vision is good [7]. Scleritis is more severe and is often associated with ocular complications. Unlike episcleritis which almostalways resolves with steroids eye drops, in about $60 \%$, scleritis will require oral corticosteroids or immunosuppressive drugs to control the inflammation [8]. The necrotising form of scleritis is the most severe and is commonly associated with RA. Scleritis that occurs in vasculitis has the worst prognosis than any other form of non-infectious diseases. Systematic review, examination and investigations are required to control severe ocular involvement. Cyclophosphamide has been reported to be the most effective immunosuppressive treatment to control severe ocular involvement [9]. It has been identified that risk factors for decreased vision in scleritis are posterior scleritis, necrotising scleritis, scleral inflammation of more than $2+$, anterior uveitis, ocular hypertension and associated infectious disease [10]. RA is the leading cause of necrotising scleritis and peripheral ulcerative scleritis both of which cause severe vision loss and can progress rapidly without treatment [11]. However scleritis and peripheral ulcerative keratitis can present before or after the onset ofRA or as an isolated condition [12]. Posterior scleritis in psoriatic arthritis with severe visual loss has been reported [13]. Progression of scleritis to the other eye can occur within five years. Associated systemic disease is seen in $50 \%$ of patients and thus a detailed medical history and physician evaluation is an important part of management [14]. Vasculitis [15]and Wegener's granulomatosis [16] as an aetiology of scleritis though rare have been reported and can cause anterior or posterior scleritis. Necrotising scleritis has been reported in association with connective tissue diseases such a scleroderma [17]. Scleritis can be acute, chronic or take a relapsing remitting course [18]. It has also been associated with diseases such as relapsing polychondritis and Vogt-Koyanagi- Harada disease [19]. In relapsing polychondritis, scleritis is bilateral, necrotising, recurrent and often requires immunomodulatory therapy for control [20]. Because of the nodular elevation of choroid sometimes, posterior scleritis has been reported to resemble a choroidal tumour in a patient with SLE [21]. Infections can also cause scleritis but it clinically appears similar to the autoimmune type. Infectious aetiology is associated with worse prognosis because of the delay in diagnosis when aggressive treatment is needed and this occurs in about $5-10 \%$ of patients with scleral inflammation. Various infections that can be bacterial, viral, fungal or parasitic have the potential to causescleritis and episcleritis. However in our study the only infection we encountered was tuberculosis which presented with nodular anterior scleritis.

\section{Conclusion}

The study on our patient population conclusively states that the incidence of systemic collagen vascular diseases is high in both episcleritis and scleritis. Visual prognosis was better in episcleritis but in those with scleritis early treatment was required to prevent complications. The association with dry eye due to collagen vascular diseases caused significant discomfort for a longer duration in both. Complete ophthalmic evaluation is required for all patients presenting with red eye as the ophthalmologist may be the first to see the patient who has an underlying collagen vascular disease which has not yet become symptomatic. It is important to differentiate betweenepiscleritis and scleritis because of the difference in the degree of complications and extent of visual loss. Episcleritis responds promptly to NSAID eye drops and the vision is not affected in these patients. Patients with mild 
diffuse or nodular scleritis respond to NSAID, those with diffuse or nodular scleritis need steroids both oral and topical and those with necrotising or nodular scleritis require immunomodualory therapy or biologics. Early detection of autoimmune or infectious underlying systemic disease allows the clinician to deliver precise management and prevent visual loss and ocular morbidity.

Funding: Nil, Conflict of interest: None initiated.

Permission from IRB: Yes

\section{References}

1. Berchicci L, Miserocchi E, Di Nicola M, La Spina C, Bandello F, ModoratiG.Clinical features of patients with episcleritis and scleritis in an Italian tertiary care referral center. Eur J Ophthalmol. 2014 May-Jun;24(3) :293-8. Doi:10.5301/ejo.5000394. Epub 2013 Nov 12.

2. Sainz de la Maza M, Molina N, Gonzalez-Gonzalez LA, Doctor PP, Tauber J, Foster CS. Scleritis therapy. Ophthalmology. 2012 Jan; 119(1):51-8. doi: 10.1016/j. ophtha.2011.07.043. Epub 2011 Oct 19..

3. Akpek EK, Thorne JE, Qazi FA, et al. Evaluation of patients with scleritis for systemic disease. Ophthalmology. 2004 Mar;111(3):501-6

4. Jabs DA, Mudun A, Dunn JP, Marsh MJ; Episcleritis and scleritis: clinical features and treatment results. Am J Ophthalmol. 2000 (Oct); 130(4):469-76

5. Hakin KN, Watson PG. Systemic associations of scleritis. IntOphthalmolClin. 1991 Summer; S31 (3):111-29.

6. Oray M, Meese H, Foster CS. Diagnosis and management of non-infectious immunemediated scleritis: current status and future prospects. Expert Rev ClinImmunol. 2016 Apr 8:1-11. [Epub ahead of print].

7. Shenoy R, Suryawanshi M, Isaac R, Philip SK. Posterior scleritis in pediatric age group: A case report and review of literature.Oman J Ophthalmol. 2016 JanApr;9(1):59-62doi: 10.4103/0974-620X. 176121

8. Carrasco MA, Cohen EJ, Rapuano CJ, Laibson PR. Therapeutic decision in anterior scleritis: our experience at a tertiary care eye center. J Fr Ophthalmol. 2005Dec; 28(10): 1065-9.
9. Ramenaden ER, Raiji VR. Clinical characteristics and visual outcomes in infectious scleritis: a review.ClinOphthalmol. 2013; 7:2113-22. doi: 10.2147/ OPTH.S37809. Epub 2013 Nov 4.

10. Sainz de la Maza M, Molina N, Gonzalez-Gonzalez LA, Doctor PP, Tauber J, Foster CSClinical characteristics of a large cohort of patients with scleritis and episcleritis. Ophthalmology. 2012 Jan; 119(1):43-50. doi: 10.1016/j.ophtha.2011.07.013. Epub 2011 Oct 2.

11. Artifoni M, Rothschild PR, Brézin A, Guillevin L, Puéchal X. Ocular inflammatory diseases associated with rheumatoid arthritis. Nat Rev Rheumatol. 2014 Feb; 10(2):108-16.doi: 10.1038/nrrheum. 2013.185. Epub 2013. Dec 10.

12. Galor A, Thorne JE. . Scleritis and peripheral ulcerative keratitis.Rheum Dis Clin North Am. 2007 Nov; 33(4):835-54, vi

13. Altan-Yaycioglu R, Akova YA, Kart H, Cetinkaya A, Yilmaz G, Aydin P. Posterior scleritis in psoriatic arthritis. Retina. 2003 Oct; 23(5):717-9.

14. Niemann KK, Bartlett JD, Heck LW Jr, McCollum CJ. Nodular scleritis: case report involving immunosuppressive therapy. J Am Optom Assoc. 1997 Dec; 68(12):782-7.

15. Castells DD. Anterior scleritis: three case reports and a review of the literature. Optometry. $2004 \mathrm{Jul}$; 75(7):430-44.

16. Sims J. Scleritis: presentations, disease associations and management. Postgrad Med J. 2012 Dec; 88 (1046):713-8.doi: 10.1136/ postgradmedj-2011130282.Epub 2012 Sep 12.

17. Nizam S, Johnstone A, Green M, Gough A. Necrotising scleritis and connective tissue disease-three cases and a review. ClinRheumatol. 2009 Mar; 28(3):339-41. Doi 10.1007/s10067-008-1054-2. Epub 2008 Dec4.

18. Cunningham ET Jr, McCluskey P, Pavesio C, Wakefield D, Zierhut M. Scleritis. OculImmunolInflamm. 2016 Feb; 24(1):2-5.doi: 10. 3109/09273948.2016.1136190. 
19. Sambhav K, Majumder PD, Biswas J. Necrotizing scleritis in a case of Vogt-Koyanagi-Harada disease.Oman J Ophthalmol. 2015 Sep-Dec; 8(3):216. doi: 10.4103/0974-620X. 169909.

20. Sainz-de-la-Maza M, Molina N, Gonzalez-Gonzalez LA, Doctor PP, Tauber J, Foster CS. Scleritis associated with relapsing polychondritis. Br J Ophthalmol. 2016 Feb 17.pii: bjophthalmol- 2015 - 306902. doi: 10.2236/bjophthalmol-2015-306902 [Epub ahead of print].

21. Sin PY, Liu DT, Young AL.Nodular Posterior Scleritis Mimicking Choroidal Tumor in a Patient With Systemic Lupus Erythematous: A Case Report and Literature Review. Asia Pac J Ophthalmol (Phila). 2015 Dec 18. [Epub ahead of print].

\section{How to cite this article?}

Mohamed AI, Annamalai R, Muthayya M. Clinical features, visual outcome and association with systemic disease in patients with episleritis and scleritis. A study of 50 eyes. Int J Med Res Rev 2016;4(5):707-712.doi: 10.17511/ijmrr.2016.i05.07. 